\title{
PER UN NUOVO CONCETTO DI GIURISDIZIONE
}

\author{
Antônio do Passo Cabral ${ }^{1}$
}

\section{RESUMEM}

L'evoluzione dello stato di diritto implica che, nella società , questioni importanti sono definite dalla magistratura. In questa linea di pensiero diventa particolarmente importante per la ricerca di un concetto di giurisdizione atual.É questo oggetto di analisi di questo articolo, al fine di analizzare i cambiamenti contemporanei di giurisdizione.

PAROLE CHIAVE: GIURISDIZIONE . MODIFICHE . MODIFICHE . SVILUPPI

\section{INTRODUZIONE}

La giurisdizione, nelle parole di Nicola Picardi, è la "vocazione del nostro tempo”. L'evoluzione dello Stato di Diritto ha fatto sì che, nella vita in società, questioni importanti vengano definite dal giudiziario. In questa linea di pensiero assume un ruolo rilevante la ricerca di un concetto di giurisdizione attuale.

Sebbene quel che si intende per giurisdizione sia in gran misura cambiato nel corso degli anni, il tema poggia su premesse che non riescono più a rispondere alle aspirazioni delle comunità umane, né riflettono la gamma multi faccettata di funzioni che gli organi giudiziari sono sollecitati ad esercitare nello Stato di Diritto contemporaneo.

In poche parole, si può dire che il mondo è radicalmente cambiato, e si continua a dire che la giurisdizione è l'attività statale che persegue la giusta composizione della lite, o che applica la legge al caso concreto.

L'obiettivo di questo breve saggio, scritto in omaggio al Professor Nicola Picardi, è quello di riprendere il tema che gli è stato molto caro ${ }^{1}$ e sottolineare alcuni aspetti dei concetti classici di giurisdizione che meritano di essere rivisti.

\footnotetext{
* Articolo scritto per il liber amicorum in omaggio all'amico Professor Avv. Nicola Picardi.

${ }^{1}$ Professor Adjunto de Direito Processual Civil da Universidade do Estado do Rio de Janeiro (UERJ), onde leciona na Graduação, Mestrado e Doutorado. Doutor em Direito Processual pela UERJ, em cooperação com a Universidade de Munique, Alemanha (Ludwig-Maximilians-Universität). Mestre em Direito Público pela UERJ. Pós-doutorando pela Universidade de Paris I (PanthéonSorbonne). Membro da Associação Internacional de Direito Processual, do Instituto Iberoamericano de Direito Processual, do Instituto Brasileiro de Direito Processual, da Associação Teuto-brasileira de Juristas (Deutsch-Brasilianische Juristenvereinigung) e da Wissenschaftliche Vereinigung für Internationales Verfahrensrecht. Procurador da República no Rio de Janeiro. Ex-Juiz Federal.
} 


\section{GIURISDIZIONE E STATALISMO. ATTIVITÀ GIURISDIZIONALE}

\section{ESERCITATA DA PRIVATI. INTERSEZIONE DELLA GIURISDIZIONE STATALE CON LE GIURISDIZIONI TRADIZIONALI.}

Una prima osservazione in relazione al concetto di giurisdizione è che dobbiamo allontanarci subito dall'idea che assimila la giurisdizione allo Stato. ${ }^{2}$ È possibile un esercizio di giurisdizione per conto di privati.

L'ascesa di alternative dispute resolutions, come l'arbitrato, è stato il primo passo per richiamare l'attenzione sugli “equivalenti giurisdizionali". ${ }^{3}$ Vogliamo fin d'ora manifestarci sul carattere giurisdizionale dell'arbitrato, senza tuttavia ignorare l'ampio dibattito in corso sulla sua natura giurisdizionale o meno. ${ }^{4} \mathrm{~A}$ nostro avviso, meno statalismo e più esistenza di un procedimento con garanzie processuali, condotto da un terzo imparziale, sarebbe la nota caratteristica che sussume all'arbitrato nel concetto di giurisdizione.

Non si può inoltre dimenticare l'esistenza di giurisdizioni tradizionali, come quelle delle comunità indigene in molti paesi, in alcuni dei quali (come la Bolivia), riconosciute come un ramo autonomo del giudiziario; in altri paesi, come la Colombia ed il Sudafrica, con intersezioni interessanti con la giustizia statale: a volte la risposta della giurisdizione indigena può esaurire o ridurre a zero la risposta della giurisdizione statale su un determinato fatto. Si vede quindi l'accettazione, da parte dell'ordinamento statale, di altre forme di soluzione di controversie nella società che, essendo equivalenti funzionali alla giurisdizione, meritano il riconoscimento e l'assorbimento nei canali formali del sistema processuale statale.

\footnotetext{
${ }^{1}$ Picardi, La giurisdizione all'alba del terzo milennio, Milano, 2007, p.1. Oltre ai testi dell'autore pubblicati in italiano, da segnalarne alcuni tradotti in portoghese e spagnolo: Jurisdição e processo, Rio de Janeiro, 2008; La Jurisdicción en el alba del tercer milenio, 2009, spec. p.9-10, 28.

${ }^{2}$ Cfr. Chiovenda, Principii di Diritto Processuale Civile. Napoli, ristampa, 1965, p.291-292; Fazzalari, Istituzioni di Diritto Processuale Civile, Padova, 8a ed., 1996, p.10-11. E che è riconosciuta come un'idea romana: Chiovenda, L'idea romana nel processo civile moderno. in Saggi di Diritto Processuale Civile, Milano, 1993, vol.3, p.79.

${ }^{3}$ Carnelutti, Istituzioni del nuovo processo civile italiano, Roma, 1941, p.55.

${ }^{4}$ Sul tema dell'arbitrato merita consulta Marino Marinelli, La natura dell'arbitrato irrituale. Profili comparatistici e processual, Torino, 2002; Bonato, La natura e gli effetti del lodo arbitrale. Studio di diritto italiano e comparato. Napoli, 2012.
} 


\section{GIURISDIZIONE NEL DIBATTITO TRA PUBBLICISMO E} PRIVATISMO. APPLICAZIONE DEL DIRITTO OGGETTIVO O GIUSTA COMPOSIZIONE DELLA LITE.

Nella culla del processualismo scientifico, dalla fine del XIX fino all'inizio del XX secolo, nell'apogeo dello Stato Liberale, si concepiva la giurisdizione volta esclusivamente all'interesse delle parti, caratterizzata da inerzia ed intesa come una funzione di tutela di una violazione del diritto materiale. Una formula nota che concettualizza la giurisdizione in modo che il risultato finale fosse più consono agli interessi delle parti, è stata la definizione di Carnelutti, focalizzata sullo scopo di ottenere "la giusta composizione della lite", 5 intesa questa come conflitto di interessi qualificato dalla pretesa dell'attore e dalla resistenza del reo. ${ }^{6}$

Con l'avvento del pubblicismo processuale comincia a delinearsi una diversa concezione della giurisdizione, fondata su interessi statali e pubblici, comunemente descritti come prevalenti su quelli di tutela del diritto delle parti. La protezione alle situazioni giuridiche processuali delle parti era conseguenza indiretta dell'applicazione della legge al caso (diritto oggettivo). In questo senso, è ormai classico il concetto di Chiovenda che considera la giurisdizione come rivelazione della volontà concreta della legge strumentalizzata dal giudice, mediante provocazione delle parti. $^{7}$

Molti autori semplicemente congiungevano o sommavano le due concezioni: giurisdizione sarebbe l'attività dello Stato che, nell'applicare la regola del diritto oggettivo, aveva come scopo la giusta composizione della lite. ${ }^{8}$

Tutto questo dibattito ha fatto sì che il concetto di giurisdizione rimanesse immerso nella concezione manichea tra pubblicismo e privatismo. Attualmente però questa dicotomia risulta superata in grande misura. Ammettere la rilevanza degli interessi privati nel processo, oppure concepire che la tutela di situazioni giuridiche soggettive sia uno scopo della giurisdizione, non significa un ritorno al privatismo romano, né porterebbe ad un'eliminazione dello Stato, la cui presenza nel rapporto

\footnotetext{
${ }^{5}$ Carnelutti, Lezioni di Diritto Processuale Civile. Padova, vol.2, 1930, p.4; Id., Istituzioni..., cit., p.38.

6 Marinoni, A Jurisdição no Estado Constitucional, in Revista da Academia Brasileira de Direito Constitucional, v. 7, 2009, p.423-514.

${ }^{7}$ In certa misura ripreso da Liebman, Manuale di Diritto Processuale Civile, Milano, vol.I, $2^{\mathrm{a}}$ ed.,1968, p. 10 .

${ }^{8}$ In Brasile fu sostenuta da Frederico Marques: “a jurisdição civil tem como causa finalis a composição justa do litígio, motivo pelo qual aplica as normas do direito objetivo, reguladoras das relações intersubjetivas, que estão na substância do conflito de interesses". Marques, Manual de Direito Processual Civil. vol.I, $13^{\mathrm{a}}$ ed., São Paulo, 1990, p.72.
} 
processuale è insopprimibile. Nel processo, gli interessi pubblici e quelli privati devono convivere armoniosamente e non esser visti come mutuamente escludenti.

\section{SEGUE. LA NECESSITÀ DI UNA COMPRENSIONE PROSPETTIVA DELLA FUNZIONE GIURISDIZIONALE}

D'altra parte, qualunque sia l'enfasi che si voglia dare, sia all'aspetto dell'applicazione dell'ordinamento oggettivo al caso, sia a quella della giusta composizione della lite, questo dibattito nasconde un altro ostacolo problematico per lo sviluppo del concetto contemporaneo di giurisdizione. $\grave{E}$ che, nelle pieghe della discussione, ha preso corpo un'immagine dell'attività giurisdizionale che si proietta fino ad oggi, un'idea che la giurisdizione attui soltanto retrospettivamente, reagendo, rispondendo alle lesioni ai diritti si et in quantum sollecitata dall'interessato. ${ }^{9}$

$\mathrm{Ci}$ si dimentica che lo Stato assume, nel mondo contemporaneo, caratteristiche molto diverse. Invece di essere un mero sanzionatore di comportamenti, lo Stato è divenuto un induttore di condotte nella società, attuando prospettivamente con l'obiettivo di fomentare e stimolare comportamenti auspicati. Questa già era l'attuazione che si intendeva necessaria nell'ambito della tutela inibitoria, svincolata da qualunque atto di lesione (attuale nemmeno potenziale). ${ }^{10} \mathrm{E}$ questa è l'attuazione che ci si aspetta in generale dal giudice nell'esercizio della funzione giurisdizionale, il che attualmente si evince dal principio del contraddittorio (inteso come diritto di influenza e dovere di dibattito $)^{11}$ e dalla cooperazione processuale. Entrambi i principi, nella loro accezione più contemporanea, permettono di imporre al giudice doveri di coinvolgimento nel dibattito, di chiarimento, di consulta, prevenzione di invalidità, ${ }^{12}$ tra molte altre funzioni che rappresentano la consacrazione di un approccio volto al futuro (future-oriented approach).

\footnotetext{
9 Anche nella tutela cautelare, l'attività giurisdizionale viene compresa come una reazione. Cfr. Carnelutti, Istituzioni, cit., p.5-7, 17; Id. Lezioni, cit., p.60-63.

${ }^{10}$ Pertanto è una modalità di tutela diversa dalla cautelare. Cfr. Mitidiero, Antecipação da tutela: da tutela cautelar à técnica antecipatória, São Paulo, $2^{\mathrm{a}}$ ed., 2014.

${ }^{11}$ Cabral, Il principio del contraddittorio come diritto d'influenza e dovere di dibattito. Rivista di Diritto Processuale, LX, n² 2, 2005, p.459 ss.

12 Didier Jr., Fundamentos do princípio da cooperação no direito processual civil português. Coimbra 2010; Oliveira, Poderes do juiz e visão cooperativa do processo. Genesis - Revista de Direito Processual Civil, $\mathrm{n}^{\circ}$ 27, 2003, p.26; Cunha, O processo civil no Estado Constitucional e os fundamentos do projeto do novo Código de Processo Civil brasileiro. Revista de Processo, v. 209, jul, 2012, p.349-374; Nunes, Processo Jurisdicional Democrático, Curitiba, 2008, p.198. In Italia Grasso, La collaborazione nel processo civile. Rivista di Diritto Processuale, XXI, n.4, 1966, p. 587.
} 
Questa è anche la portata che si esige dal giudiziario nella gestione della sicurezza giuridica contemporanea. Qualche anni fa gli studiosi del diritto pubblico si sono accorti del bisogno di garantire sicurezza verso il futuro. Nel processo civile questo sviluppo fa con che il superamento di qualunque modalità di stabilità processuali (preclusioni, cosa giudicata, v.g.per forza di rimessione in termine o revocatoria), sia coniugata con regole di transizione pronunciate dal giudice per ridurre l'impatto della rottura di un regolamento stabile ed il passaggio ad un nuovo. ${ }^{13} \mathrm{Si}$ tratta di un altro esempio di attività giurisdizionale prospettiva, e non retrospettiva.

\section{SOSTITUIBILITÀ COME CARATTERISTICA DELLA GIURISDIZIONE. LA GIURISDIZIONE VOLONTARIA ED IL CRESCENTE SPAZIO PER L'AUTOREGOLAZIONE FORMALE}

Molti autori sparsi nel mondo indicano la sostituibilità come caratteristica della giurisdizione. ${ }^{14}$ Lo Stato (il giudice) agirebbe per sostituire con la sua, la volontà dei litiganti in conflitto. Difatti, nell'assumere il monopolio dell'esercizio della giurisdizione, lo Stato proibisce l'autotutela, riducendo la possibilità che l'individuo, con l'uso unilaterale della volontà o della forza, possa sottomettere altri individui alla sua pretesa. In questo scenario, la volontà statale, nel definire come deve esser risolto il conflitto, sostituirebbe la volontà dei litiganti. Questa supposta caratteristica della giurisdizione ha fatto sì che alcuni tipi di attività giudiziaria non venissero considerati tipicamente giurisdizionali. È quel che è avvenuto con la cosiddetta giurisdizione volontaria, considerata tradizionalmente come un'attività amministrativa semplicemente perché andrebbe esercitata anche se gli interessati sono concordanti. ${ }^{15}$ Per questo motivo alcuni la definiscono come attività "jus-integrativa": la volontà degli individui non è sufficiente per garantir loro 1'effetto giuridico preteso, essendo necessario l'avallo giudiziale. Quindi, siccome la volontà espressa dallo Stato è emessa in senso convergente con la volontà dei litiganti, non vi sarebbe esattamente sostituibilità, e quindi quell'attività non potrebbe esser considerata giurisdizionale. Orbene, consideriamo un equivoco immaginare che tutta l'attività giurisdizionale debba essere

\footnotetext{
${ }^{13}$ Cabral, Coisa julgada e preclusões dinâmicas: entre continuidade, mudança e transição de posições processuais estáveis, Salvador, $2^{\mathrm{a}}$ ed., 2014, p.559 ss.

${ }^{14}$ Chiovenda, Principii, cit., p.296.

${ }^{15}$ Cfr. Carnelutti, Lezioni, cit., p.139; Chiovenda, Principii, cit., p.314-317; Fazzalari, Istituzioni, cit., p.557.
} 
sostitutiva della volontà delle parti. Se così fosse, se il Pubblico Ministero, nel processo penale, chiedesse l'assoluzione dell'accusato, si verificherebbe una giurisdizione volontaria?

Inoltre l'idea soggiacente alla giurisdizione volontaria è quella di un paternalismo che sembra incompatibile con le società attuali. Ci sembra che stiamo già vivendo in un'era di palese declino della giurisdizione volontaria, e in un futuro prossimo è probabile che questo tipo di procedimento si vada riducendo fino alla sua quasi completa eliminazione.

Bisogna infine sottolineare ancora una chiara tendenza contemporanea: quella di fomentare l'autocomposizione e conferimento di poteri ai litiganti, visto che aumentano sempre più gli spazi in cui la loro volontà è sufficiente per produrre effetti giuridici. In questo senso è in crescita anche l'autonomia delle parti nel definire persino le regole del procedimento che il giudice deve applicare. Gli accordi o convenzioni processuali, che sempre più vengono popolarizzati e adottati nella prassi e nella legislazione comparata (p.es. l'art.190 del CPC brasiliano del 2015), sono la maggior espressione dell'accettazione dell'autoregolamento della volontà. ${ }^{16}$ Non solo nell'arbitrato, oppure attraverso la conciliazione o la mediazione extragiudiziali - dove l'autocomposizione sui diritti sostanziali era ormai riconosciuta ${ }^{17}$ - ma anche nel processo statale, le parti possono definire le regole processuali applicabili al caso. In questo contesto sarebbe corretto dire che il giudice applica la regola legale? $O$ che la giurisdizione corrisponderebbe formalmente ad un'attività etero compositiva o sostitutiva?

\section{NATURA DICHIARATORIA DELLA GIURISDIZIONE. L'ATTIVITÀ GIURISDIZIONALE COME DICHIARAZIONE DI DIRITTI PREESISTENTI. MONISMO E DUALISMO NELLA LETTERATURA CLASSICA.}

Un'altra caratteristica comunemente indicata nella dottrina tradizionale è la natura dichiaratoria della giurisdizione. ${ }^{18}$ Questa concezione si basa sul dualismo dell'ordinamento giuridico: i diritti soggettivi preesistono alla sentenza, che non avrebbe nessun carattere creativo. L'attività del giudice si limiterebbe a pronunciare o rivelare l'esistenza di diritti nell'ordinamento oggettivo.

\footnotetext{
${ }^{16}$ Cabral, Convenções processuais entre publicismo e privatismo, São Paulo, 2015.

${ }^{17}$ Carnelutti, Istituzioni, cit., p.55; Id. Lezioni, cit., p.87-96. Chiovenda, d'altra parte, sosteneva che la conciliazione appartiene alla giurisdizione volontaria. Cfr. Chiovenda, Principii, cit., p.322-323.

${ }^{18}$ Chiovenda, Principii, cit., p.179; Carnelutti, Istituzioni, cit., p.27.
} 
Mentre i difensori del monismo immaginavano che i diritti fossero effettivamente creati dalla sentenza. Prima della sentenza esisterebbero soltanto interessi materiali. È evidente che questa concezione sbarra nel concetto di giurisdizione. Salvatore Satta, e.g., si rivolse con veemenza contro quel che denominava una "giurisdizione statica $e$ passiva”. Per lui, la giurisdizione non si poteva ridurre a dichiarare il diritto previsto dalla legge. Si doveva rompere il dualismo diritto oggettivo/diritto soggettivo e la concezione dualistica dell'ordinamento giuridico. Nell'adottare una concezione unitaria dell'ordinamento giuridico, la giurisdizione assumerebbe un carattere creativo del diritto (jus dicere). ${ }^{19}$

Perduto in questo dibattito tra dualismo e monismo, il concetto di giurisdizione rimaneva ostaggio delle ripercussioni possibili dell'adozione dell'una o dell'altra premessa.

7. SEGUE. SVILUPPO IN RELAZIONE A QUESTA CONCEZIONE. LA GIURISDIZIONE COME ATTIVITÀ PARZIALMENTE CREATIVA DELLA NORMA. LA FUNZIONE NON PURAMENTE DICHIARATIVA DI EFFETTIVAZIONE DI DIRITTI. NECESSARIA RIMOZIONE DEL CONCETTO DI DIRITTO SOGGETTIVO: PROCEDIMENTI PROBATORI ANTE CAUSAM E LA FUNZIONE DI CONSULTA.

Attualmente i dibattiti più attuali nella teoria del diritto e nella teoria delle norme ormai ci chiariscono che l'attività giurisdizionale non è solo dichiarativa di un senso previo che la norma già possieda. La giurisdizione è anche in una certa misura creativa, costruendo o ricostruendo il senso della norma nello stesso procedimento della sua interpretazione ed applicazione. D'altra parte, la rivalutazione della giurisprudenza come fonte di diritto, come si evince dalla vincolabilità che i precedenti giurisprudenziali hanno acquisito, mostra anche un altro sviluppo di questa stessa tendenza. ${ }^{20}$

Sconfitto il preconcetto, dovremmo vedere concetti di giurisdizione sempre più lontani da una supposta natura dichiaratoria che non si verifica, ridimensionando anche il ruolo della tutela dichiaratoria, volta non alla rivelazione di diritti, ma intesa come una maniera per rimuovere uno stato di incertezza.

\footnotetext{
${ }^{19}$ Satta-Punzi, Diritto Processuale Civile, Padova, $13^{\mathrm{a}}$ ed., 2000, p.10-12.

${ }^{20}$ Zaneti Jr., $O$ valor vinculante dos precedentes. Salvador, 2014. C'è versione italiana della tese di dottoramento presentata all'Università degli Studi di Roma Tre nel 2014: "Il valore vincolante dei precedenti”.
} 
E, di fatto, alcuni avanzamenti di questa riflessione già si possono percepire nella dottrina processuale. Si comincia a riconoscere che la funzione dichiarativa di diritti non esaurisce le molteplici funzioni giurisdizionali. Il giudice è costantemente chiamato a riparare la trasgressione a quanto prescritto in una norma giuridica (applicando le ulteriori misure di riparazione o di sanzione previste dall'ordinamento) o a realizzare praticamente e concretamente una situazione di vantaggio. ${ }^{21}$

Ma anche negli autori più contemporanei, sembra esistere ancora un necessario legame della giurisdizione al concetto di diritti soggettivi. Nel nostro parere, esiste un campo più ampio in cui le persone possono aver interesse a definire giudizialmente un certo aspetto di una situazione giuridica, per la sicurezza che gli offrirebbe l'attività giurisdizionale, indipendentemente che tale utilità, degna di tutela, corrisponda ad un effettivo diritto soggettivo. A volte l'attività giurisdizionale serve esattamente affinché la parte sappia se ha o non ha un certo diritto.

Ricordiamo qui i procedimenti probatori ante causam, già tanto studiati nella dottrina mondiale ${ }^{22}$ (ed ora incorporati nel Codice di Processo Civile Brasiliano del 2015, art.381), che hanno lo scopo di evitare il litigio mediante la rivelazione fattuale. Come immaginare che un procedimento di certificazione fattuale si inquadri pienamente in alcuni dei concetti di giurisdizione tradizionali?

Bisogna sottolineare anche la funzione di consulta, mediante la quale gli individui ricorrono al organo giudiziario per ottenere un'interpretazione del diritto soggettivo. Questa funzione (che in Brasile esiste nei tribunali elettorali), si distacca visibilmente dall'esistenza di un conflitto di interessi e perfino dall'esistenza di un diritto soggettivo. L'interessato va dal giudice per ottenere una risposta statale che orienterà la sua condotta futura riguardo a quella situazione. A partire dalla riposta alla consulta, la parte potrà anche comprendere che non ha nessun diritto soggettivo, e quindi rinunciare ad esercitare una pretesa che, se fatta valere, potrebbe esser considerata infondata.

\section{DEFINITIVITÀ. LA COSA GIUDICATA COME MERAMENTE ACCIDENTALE ALL'ATTIVITÀ GIURISDIZIONALE}

\footnotetext{
${ }^{21}$ Didier Jr., Curso de Direito Processual Civil. vol.I, Salvador, 17ª ed, 2015, p.153: "a jurisdição é a função atribuída a terceiro imparcial de realizar o direito de modo imperativo e criativo (reconstrutivo), reconhecendo/efetivando/protegendo situações jurídicas concretamente deduzidas, em decisão insuscetivel de controle externo e com aptidão para tornar-se indiscutivel".

${ }^{22}$ Besso, La prova prima del processo, Torino, 2004; Yarshell, Antecipação da prova sem o requisito da urgência e direito autônomo à prova, São Paulo, 2009.
} 
È ancora molto comune associare la cosa giudicata alla funzione giurisdizionale. Molti autori affermano che è nell'essenza della giurisdizione l'esistenza della cosa giudicata o almeno l'attitudine delle decisioni giurisdizionali alla definitività da essa decorrente (immutabilità e indiscutibilità).

Discordiamo da questo pensiero. Come già affermato in un altro saggio, la cosa giudicata non è essenziale allo Stato di Diritto e nemmeno alla giurisdizione. ${ }^{23}$ Vi sono varie decisioni giurisdizionali che non acquisiscono una stabilità tanto forte come la cosa giudicata, ma non per questo perdono il loro carattere giurisdizionale. D'altra parte, bisogna ricordare che vi sono varie forme di stabilità processuale differenti dalla cosa giudicata: è un errore pensare alla cosa giudicata come l'unica stabilità degli atti processuali o anche delle sentenze, ed è anche sorpassato fondare il sistema delle stabilità su un manicheismo per il quale o abbiamo la cosa giudicata o l'atto processuale non ha alcuna stabilità. ${ }^{24}$ In molti ordinamenti, ed in altri campi, come il diritto costituzionale e amministrativo, già si percepisce che si può raggiungere la stabilità degli atti giuridici preteriti senza che la protezione del loro contenuto da alterazioni posteriori avvenga necessariamente mediante la cosa giudicata.

Se è così persino per le sentenze che risolvono il merito, bisogna ricordare anche la tutela provvisoria, di carattere cautelare o soddisfattorio, che non comprende esattamente nessuna dichiarazione di diritti che tenda alla definitività anccorchè alcuni dei suoi effetti possano acquisire stabilità.

\section{BREVE CONCLUSIONE}

Nicola Picardi è sempre stato un uomo all'avanguardia del suo tempo. La sua opera ha gettato nuova luce sulle funzioni dello Stato e del processo nel mondo contemporaneo, lanciando le basi affinché, non solo in Italia, ma nel resto del mondo, innumerevoli colleghi ed amici tornassero al tema della giurisdizione, cercando di concettualizzarla sotto l'ottica delle nuove funzioni che il giudiziario è chiamato ad esercitare.

\footnotetext{
${ }^{23}$ Cabral, Coisa julgada e preclusões dinâmicas: entre continuidade, mudança e transição de posições processuais estáveis, cit., p.267 ss, 274 ss.

${ }^{24}$ Cabral, Coisa julgada e preclusões dinâmicas: entre continuidade, mudança e transição de posições processuais estáveis, cit., p.294 ss.
} 
Lo scopo di questo breve saggio non è quello di dare risposte definitive, ma appena di accennare ad alcuni aspetti del concetto di giurisdizione che riteniamo meritino una rivisitazione da parte degli studiosi del diritto processuale. 